\title{
Anomaly Detection for Diagnosing Failures in a Centrifugal Compressor Train
}

\author{
Ian PALACÍN ${ }^{\natural}$ Daniel GIBERT ${ }^{\natural}$ Jordi PLANES』1, Simone ARENA ${ }^{\natural}$, \\ Pier Francesco ORRUं ${ }^{\natural}$, Maurizio MELIS ${ }^{\text {[ }}$, and Marco ANNIS ${ }^{\square}$ \\ ${ }^{a}$ Universitat de Lleida \\ ${ }^{\mathrm{b}}$ Università degli Studi di Cagliari \\ ${ }^{\mathrm{c}}$ SARLUX srl
}

\begin{abstract}
Predicting machine failures is of the utmost importance in industrial systems as it can turn expensive crashes and repair costs into affordable maintenance costs. To this end, this paper presents preliminary work for detecting failures in a centrifugal compressor train based on sensorial data. We show the detection capabilities of a two-step process consisting of: (1) a preprocessing step to reduce the dimensionality of the input data using Principal Component Analysis, and (2) an anomaly detection step using the Mahalanobis distance to detect anomalous observations on the sensors' data. The experiments using real-world data demonstrate the feasibility of our approach and the ability of the method to detect the failures eight days in advance.
\end{abstract}

Keywords. failure detection, centrifugal compressor, feature reduction, anomaly detection

\section{Introduction}

During the lifetime of any industrial machine, its components might eventually break, producing an expensive machine breakdown [3]. Thus, diagnosing and detecting machine failures before they occur is essential to avoid the possibility of catastrophic failures, save costs, and keep the machinery running for longer periods of time. Traditionally, the diagnosis of failures in industrial machinery relied on human-expert criteria for the development of an expert system, modeling the expertise knowledge of an expert or group of experts. However, such systems may fail for various reasons including the unavailability of experts and the difficulty to translate the expert knowledge into a concrete method, among others [1].

This increasing complexity of modern machinery aggravates the quality of diagnosis, forcing a shift from a model-based approach to a data-based approach for equipment monitoring and diagnose of failures [11]. As a result, collecting operating and sensor information, from a machine while it is working, has become of the utmost importance

\footnotetext{
${ }^{1}$ Correspondence to: J. Planes. INSPIRES Research Centre, University of Lleida. C/Jaume II, 69. Lleida, Spain. Tel.: +34 973702764; E-mail: jordi.planes@udl.cat.
} 
to detect anomalies that might manifest a future component failure. Various approaches have been presented in the literature [40|6/10] that employ different signal sources to detect failures of industrial machinery, including vibration signals, acoustic emission signals, temperature, and electrical parameters, among others. For instance, Z. Hai-Yang et al. [4] presented a method for diagnosing failures in a reciprocating compressor based on features extracted from vibration signals using Local Mean Decomposition (LMD) and Multi-Scale Entropy (MSE) methods. Y. Wang et al. [9] used acoustic emission signals coupled with the simulated valve motion to diagnose faults in reciprocating compressor valves. In addition, due to the strong correlation and high dimensionality of the data obtained from the sensors installed in complex machinery, dimensionality reduction techniques such as Principal Components Analysis (PCA), have been applied in the literature in order to reduce the number of input variables used to model the data [610].

In this paper, a two-step approach to detect failures in a centrifugal compressor train is presented, which relies on the data provided from its sensors. The first step involves the preprocessing of the input data using Principal Component Analysis to reduce its dimensionality. Afterwards, outliers are detected using the Mahalanobis distance in the second step. To evaluate our approach, historical data (throughout 5 years) of the centrifugal compressor train has been used, including temperature, pressure, and vibration of the system valves.

The rest of the paper is organized as follows. Section 2 details the two-step approach presented in this paper. Section 3 presents various experiments used to validate the feasibility of our approach. Lastly, Section 4 presents the final remarks of the paper.

\section{Methodology}

In this section, we present a two-step process to detect failures in a centrifugal compressor train. The information is provided by 45 sensors distributed along the three main components of the centrifugal compressor train, i.e. the electric motor, the gearbox, and the compressor. The sensors are of five different types: 13 for vibration, 3 for axial displacement, 25 for temperature, 4 for pressure, and 2 for flow. Each sensor may have a different time granularity. The two-step process is as follows:

1. Principal Component Analysis (PCA) is applied to the sensor data in order to reduce the dimensionality of the strongly correlated input variables [7]. PCA provides an orthogonal projection of the data into a lower dimensional linear space, known as the principal subspace, such that the variance of the projected data is maximized [2]. The method allows to select a small number of principal components of the projection. When applied to sensors with different granularity, the coarsest granularity has been chosen.

2. Mahalanobis distance [8] is the multivariate form of the distance measured in units of standard deviation. As outliers are observations that deviate from the global behavior of the majority of data, the usage of the Mahalanobis distance allows to quantify the closeness between the current observation and their distribution and thus, detect when the behavior of the system is anomalous. 


\section{Experimentation}

To evaluate the viability of our two-step process for detecting failures, historical data of a multistage centrifugal compressor for oil and gas service has been used. This data consists of temperature, pressure and vibration of the system monitored with the sensors through five years. In all, ten failures of the compressor were provided. We present three experiments (cf. Figures $1 \mathrm{a}, 1 \mathrm{~b}$ and $1 \mathrm{c}$ ) to visualize our two-step process.

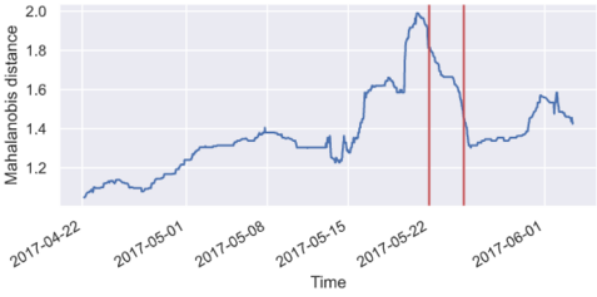

(a) Failure on the 22/05/2017. Sensors: 2 axial displacement. Component: compressor.

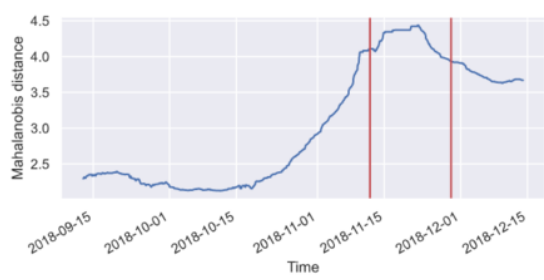

(b) Failure on the 12/11/2018. Sensors: 3 vibration, 3 temperature. Component: electric motor.

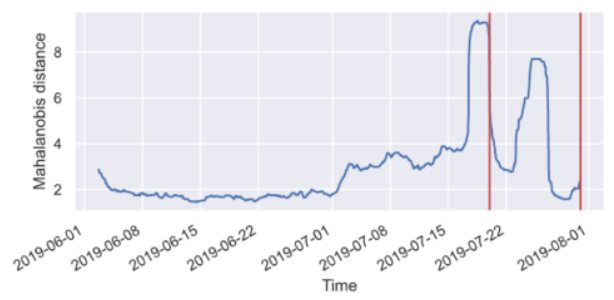

(c) Failure on the 20/07/2019. Sensors: 3 vibration, 3 temperature. Component: electric motor.

Figure 1. Mahalanobis distance for several sensors in the compressor.

The figures represent the Mahalanobis distance of the data taken at different periods of time, and the red vertical bars represent the periods were failures were reported: Figure 1a shows a failure with a valve in the compressor from 22/05/2017 to 25/05/2017; Figure $1 \mathrm{~b}$ shows a failure with a valve that started on $12 / 11 / 2018$ and ended on 29/11/2018; and Figure $1 \mathrm{c}$ shows a failure occurred with an oil filter from $20 / 07 / 2019$ to $31 / 07 / 2019$. The initial features used to detect the different failures may vary between one failure and another, and were selected using expert knowledge of the system, i.e. some features are more useful than others to detect some of the failures. For instance, the data in Figure $1 \mathrm{a}$ comes from the axial displacement of the compressor, whereas Figures $1 \mathrm{~b}$ and $1 \mathrm{c}$ use temperature and vibration information of the electric motor of the compressor train, respectively.

Before the time period of any failure, it can be observed that the Mahalanobis distance dramatically increases, reaching its peak in the failure. This could be due to the degradation of some components in the machine, what may cause the failure. A machine component usually enters into an abnormal state before its failure [5]. In most cases the anomalies were noticeable 8 to 10 days before the failure, providing a good indicator of the feasibility of the Mahalanobis distance to predict failures. 


\section{Conclusion and Future Work}

In order to diagnose failures in a compressor, we have analyzed a two-step process consisting of a feature preprocessing step and an outlier detection step. The process has been evaluated with real-world data, consisting of five years of historical data of a centrifugal compressor train. Results show that our approach may be used to predict failures in industrial systems. Particularly in our case, it can detect anomalies eight days in advance.

A future line of research would be the application of neural networks autoencoders for the anomaly detection in order to automatically trigger the corresponding warnings when the anomalies were detected.

Acknowledgments This work was partially funded by Spanish Project PID2019111544GB-C22, by the European Union's Horizon 2020 Research and Innovation Programme under Grant Agreement 723596 and Grant Agreement 768824, and by 2017 SGR 1537.

\section{References}

[1] M. Z. Bell. Why expert systems fail. Journal of the Operational Research Society, 36(613619), 1985.

[2] C. M. Bishop. Pattern Recognition and Machine Learning (Information Science and Statistics). Springer-Verlag, Berlin, Heidelberg, 2006.

[3] H. Groenevelt, L. Pintelon, and A. Seidmann. Production lot sizing with machine breakdowns. Management Science, 38(1):104-123, 1992.

[4] Z. Hai-yang, W. Jin-dong, X. Jun-jie, and G. Yi-qi. A feature extraction method based on lmd and mse and its application for fault diagnosis of reciprocating compressor. Journal of Vibroengineering, 17(7):3515-3526, 2015.

[5] D. Jiang and C. Liu. Machine condition classification using deterioration feature extraction and anomaly determination. IEEE Transactions on Reliability, 60(1):41-48, 2011.

[6] Y. Jiang, B. He, P. Lv, J. Guo, J. Wan, C. Feng, and F. Yu. Actuator fault diagnosis in autonomous underwater vehicle based on principal component analysis. In 2019 IEEE Underwater Technology (UT), pages 1-5, 2019.

[7] I. T. Jolliffe. Principal Component Analysis. Springer-Verlag, Berlin; New York, 1986.

[8] Sprnger, editor. Mahalanobis Distance, pages 325-326. Springer New York, New York, NY, 2008.

[9] Y. Wang, C. Xue, X. Jia, and X. Peng. Fault diagnosis of reciprocating compressor valve with the method integrating acoustic emission signal and simulated valve motion. Mechanical Systems and Signal Processing, 56-57:197-212, 2015.

[10] Y. Yinghua, S. Guoqiang, and S. Xiang. Fault monitoring and classification of rotating machine based on pca and knn. In 2018 Chinese Control And Decision Conference (CCDC), pages 1795-1800, 2018.

[11] S. M. Zanoli, G. Astolfi, and L. Barboni. Applications of fault diagnosis techniques for a multishaft centrifugal compressor. In 18th Mediterranean Conference on Control and Automation, MED'10, pages 64-69, 2010. 